\title{
Effect Of XBP1 Deficiency In Cartilage On The Regulatory Network Of LncRNA/circRNA-miRNA- mRNA
}

\author{
Xiaoli Li1 ${ }^{1}$ Yuyou Yang1, Li Liang11, Mengtian Fan¹, Xingyue Li1, Naibo Feng1, Yiming Pan¹, Qiaoyan Tan², \\ Qingbo $\mathrm{Xu}^{3}$, Yangli Xie ${ }^{2}$, Fengjin Guo ${ }^{1 凶}$ \\ 1. Department of Cell Biology and Genetics, Core Facility of Development Biology, Chongqing Medical University, Chongqing 400016, China. \\ 2. Department of Wound Repair and Rehabilitation Medicine, State Key Laboratory of Trauma, Burns and Combined Injury, Trauma Center, Research \\ Institute of Surgery, Daping Hospital, Army Medical University, Chongqing 400042, China. \\ 3. Cardiovascular Division, King's College London BHF Centre, London, United Kingdom. \\ $\triangle$ Corresponding author: Fengjin Guo, Department of Cell Biology and Genetics, Core Facility of Development Biology, Chongqing Medical University, \\ Chongqing, 400016, China. E-mail: guo.fengjin@gmail.com; guo.fengjin@cqmu.edu.cn.
}

(c) The author(s). This is an open access article distributed under the terms of the Creative Commons Attribution License (https://creativecommons.org/licenses/by/4.0/). See http://ivyspring.com/terms for full terms and conditions.

Received: 2021.06.19; Accepted: 2021.10.29; Published: 2022.01.01

\begin{abstract}
X-box binding protein $1(\mathrm{XPP})$ is a critical component for unfolded protein response (UPR) in ER stress. According to previous studies performed with different $X B P I-$ deficient mice, the $X B P I$ gene affects mouse cartilage development and causes other related diseases. However, how the complete transcriptome, including mRNA and ncRNAs, affects the function of cartilage and other tissues when $X B P I$ is deficient in chondrocytes is unclear. In this study, we aimed to screen the differentially expressed (DE) mRNAs, circRNAs, IncRNAs and miRNAs in XBPI cartilage-specific knockout (CKO) mice using high throughput sequencing and construct the circRNA-miRNA-mRNA and IncRNA-miRNA-mRNA regulatory networks. DE LncRNAs (DE-LncRNAs), circRNAs (DE-circRNAs), miRNAs (DE-miRNAs), and mRNAs [differentially expressed genes (DEGs)] between the cartilage tissue of XBPI CKO mice and controls were identified, including 441 DE-LncRNAs, 15 DE-circRNAs, 6 DE-miRNAs, and 477 DEGs. Further, 253,235 IncRNA-miRNA-mRNA networks and 1,822 circRNA-miRNA-mRNA networks were constructed based on the correlation between IncRNAs/circRNAs, miRNAs, mRNAs. The whole transcriptome analysis revealed that XBPI deficiency in cartilage affects the function of cartilage and other different tissues, as well as associated diseases. Overall, our findings may provide potential biomarkers and mechanisms for the diagnosis and treatment of cartilage and other related diseases.
\end{abstract}

Key words: XBP1, whole transcriptome analysis, cartilage, circRNA-miRNA-mRNA, IncRNA-miRNA-mRNA

\section{Introduction}

Abnormal chondrocyte differentiation is an important cause of common orthopedic diseases, such as rickets, dwarfism, and osteoarthritis [1,2]. During endoplasmic reticulum stress, IRE1a undergoes autophosphorylation and activation, and the XBP1u mRNA is spliced in the cytoplasm to produce the transcription factor, $\mathrm{XBP} 1 \mathrm{~s}$, with multiple regulatory functions. This transcription factor enters the nucleus to regulate the transcription and expression of its associated gene, and participates in several pathophysiological processes by regulating cell growth and apoptosis, differentiation and development, immune response, among others [3-6]. The unfolded protein response (UPR) signaling pathway, inositol-requiring enzyme 1 (IRE1)-XBP1, which is mediated by XBP1s, is an important pathway in the determination of cell fate and a key pathway for plasma cell differentiation [3-5]. According to our previous studies, XBP1s is expressed in the proliferation and hypertrophy stages of chondrocyte differentiation and is involved in the regulation of chondrocyte differentiation and cartilage development in chondrocytes. As a BMP2-inducible transcription factor, $\mathrm{XBP} 1 \mathrm{~s}$ positively regulates 
endochondral bone formation by activating GEP chondrogenic growth factor [6]. Cameron et al. reported that the cartilage-specific deficiency of XBP1 in mouse leads to a chondrodysplasia characteristically defined by reduced chondrocyte proliferation and delayed cartilage maturation and mineralization. XBP1 was inferred to control chondrocyte proliferation and the timing of cartilage maturation and matrix mineralization during endochondral ossification $[7,8]$. As reported in many literatures, in eukaryotic genomes that transcribe up to $90 \%$ of genomic DNA, only $1-2 \%$ of the transcripts encode proteins, with most being transcribed as non-coding RNAs (ncRNAs). The number of ncRNAs increases significantly with the complexity of the organism. Although the expression level of most predicted ncRNAs is markedly lower than that of mRNAs, they do not affect their regulatory functions. Based on increasing evidence, ncRNA has a regulatory role in the development process and in the response to stress and environmental stimuli [9-14].

Non-coding RNAs play an important regulatory role in cells. In fact, these RNAs, can regulate several biological processes, including epigenetic modification, variable RNA splicing, and protein stability [15-17]. For instance, rRNAs, tRNAs, small nuclear RNA (snRNAs), small nucleolar RNAs (snoRNAs) are involved in mRNA translation, splicing, modification of rRNAs, respectively [10]. High-throughput sequencing (NGS) technology and bioinformatics analysis have led to the discovery of a remarkable number of circRNAs, lncRNAs, miRNAs, mRNAs and their networks [18]. Long-noncoding RNAs (LncRNAs) are ncRNAs longer than $200 \mathrm{nt}[19]$. Previous studies have shown that lncRNAs play a crucial role in the regulation of disease processes and gene expression [20, 21]. Further, lncRNAs are reported to participate in cell differentiation, migration, proliferation and apoptosis [22]. For example, a novel osteogenesis-associated lncRNA (lncRNA-OG) significantly promotes BM-MSC osteogenesis. LncRNA-OG interacts with heterogeneous nuclear ribonucleoprotein $\mathrm{K}$ (hnRNPK) protein to regulate the activation of the bone morphogenetic protein signaling pathway. hnRNPK positively regulates the transcriptional activity of lncRNA-OG by promoting H3K27 acetylation of the IncRNA-OG promoter [23]. CircRNAs is a class of ncRNAs widely expressed in eukaryotic cells. CircRNAs have a covalent closed circular configuration without $5^{\prime}$ and $3^{\prime}$ polarities and poly A tail structure [24]. CircRNA can act as an miRNA sponge to affect the functions of miRNAs [25], and has a regulatory effect on several diseases, such as cancer, diabetes, cardiovascular diseases and certain immune diseases [26]. MiR-124-3p is an essential microRNA (miRNA), and its expression changes are related to the proliferation ability of bone mesenchymal stem cells (BMSCs) [27]. MiR-124-3p is also reported to be associated with osteoporosis (OP) and fragility fractures [28]. RNAs have become a novel hotspot in research in cartilage development. Although some ncRNAs, including lncRNAs, circRNAs and miRNAs, have been identified in bone development and associated diseases, the ncRNAs related to the regulation of XBP1 have not been revealed.

The coordinated regulation between different tissues and organs is performed by the full spectrum of transcriptome RNAs, including mRNAs and ncRNAs. In the current study, we attempted to elucidate whether and how XBP1 deficiency affects the full spectrum of ncRNA functional characterization and the normal physiological functions of ncRNA and their role in human diseases. First, we conducted whole transcriptome sequencing of cartilage tissue obtained from XBP1 conditional knockout mice, and carried out a differential expression profile assay of circRNAs, lncRNAs, miRNAs and mRNAs. Thereafter, based on bioinformatics analysis, two competitive endogenous RNA (ceRNA) regulatory networks (the circRNA-miRNA-mRNA and lncRNAmiRNA-mRNA) were established, and GO and KEGG enrichment analyses were performed.

XBP1 deficiency in cartilage leads to differences in the complete transcriptome RNA of cartilage and other different tissues, including various ncRNAs with differential expression and regulatory profiles, as well as differences in protein expression and abnormalities of various different secreted factors. Our results support the identification of both the full spectrum of the function and characterization of ncRNAs induced by XBP1 deficiency in cartilage and the role of these XBP1-related ncRNAs in normal physiological functions and human diseases.

\section{Materials and Methods}

\section{Animals and Cartilage Tissue Collection}

The XBP1 flox/flox mice was a gift from Prof. Qingbo $\mathrm{Xu}$ (King's College London BHF Centre, London, United Kingdom), the Col2 Cre mice was a gift from Prof. Lin Chen (Third Military Medical University; Chongqing, China) $[29,30]$. The primer is the following: XBP1 flox/flox-F: 5'-TGG CAA GGC TGA GCC TGA TCG-3'; XBP1 flox/flox_R: 5'-GGA ACTAGAGATACC ACTGAG-3' (XBP1 flox/flox 315 bp; $\mathrm{XBP}^{\text {flox} /+}$ 265bp and $315 \mathrm{bp} ; \mathrm{XBP}^{+/+} 265 \mathrm{bp}$ ); col2Cre-F: 5' - GAGGGTCCAGCCCGAGCTACTT-3', col2CRE-R: 5'-GCATCGACCGGTAATGCA GGC-3'. All the animal studies were performed in accordance 
with Chongqing Medical University Institutional Animal Care and Use Committee. Mice were group housed $\left(25^{\circ} \mathrm{C}\right)$ on a 12-hour light-dark cycle. All mice were maintained on a C57BL/6J background; genotyping of $\mathrm{XBP} 1^{\text {flox/flox }}$ col2 $\mathrm{CRE}^{+}(\mathrm{CKO})$ mice and control mice (XBP1 flox/flox col2CRE-) littermates was performed as described previously [29-31]. Three pairs of littermates (XBP1 flox/flox col2CRE ${ }^{+}$and $\mathrm{XBP} 1^{\text {flox/flox }}$ col2CRE-) were sacrificed at 4 weeks and hyaline cartilage tissues were processed for full transcriptome RNA-seq.

\section{Total RNA Extraction and Quality Control}

Total RNA from 6 samples was extracted using the TRIzol reagent (Invitrogen, Carlsbad, CA, USA). The concentration of total RNA was checked by using Qubit ${ }^{\circledR}$ RNA Assay Kit in Qubit ${ }^{\circledR} 2.0$ Fluorometer, its purity was checked by using a NanoPhotometer spectrophotometer (IMPLEN, CA, USA), and its integrity was checked by Bioanalyzer 2100 system (Agilent Technologies, CA, USA).

\section{RNA-seq Library Preparation and Sequencing}

For constructing the library of mRNA or circRNA, $5 \mu \mathrm{g}$ of RNA for per sample was used as input. For mRNA library construction, Epicentre Ribo-Zero ${ }^{\mathrm{TM}}$ rRNA Removal Kit (Illumina, USA) was used to remove rRNA from the total RNA. For circRNA library construction, $3 \mathrm{U}$ of RNase $\mathrm{R}$ (Epicentre, USA) per microgram was added to the rRNA removal system and incubated to remove linear RNA. Subsequently, the sequencing libraries were prepared in accordance with by using NEBNext ${ }^{\circledR}$ Ultra $^{\mathrm{TM}}$ Directional RNA Library Prep Kit for Illumina ${ }^{\circledR}$ (NEB, USA). The Agilent Bioanalyzer 2100 system was used to detect the quality of the RNA library. After the library was qualified, the Illumina PE150 sequencing was performed. Considering that the Ribo-Zero library contains both mRNA and lncRNA, we will select lncRNA by establishing a series of strict screening conditions in the data analysis part. For miRNA library construction, $5 \mu \mathrm{g}$ of total RNA for per sample was used as input using the NEBNext@ Multiplex Small RNA Library Prep Set for Illumina ${ }^{\circledR}$ (NEB, USA) according to the manufacturer's protocol. Similarly, library quality was detected on the Agilent Bioanalyzer 2100 system. the Illumina PE150 sequencing was performed.

\section{Data Analysis}

The libraries were sequenced using an Illumina sequencing platform. Then adapter sequences and reads with poor quality were filtered using FASTX toolkit pipeline (version 0.0.13). Clean reads were aligned to the UCSC (mus_musculus_Ensembl_97) using Hisat2 (http://ccb.jhu.edu/software/hisat2)
[10]. For transcriptome assembly, the mapped reads of each sample were assembled by StringTie (v1.3.1) [32], which has specific parameters for different libraries, and can accurately splice transcripts then achieve transcript quantification [33]. Then, GC contents, Q20 and Q30 of the clean data were calculated.

We use soft of find_circ [34] and CIRI2 [35] to detect and identify circRNA.The workflow of find_circ is as follows: 1) The reads that are continuously aligned with the genome are filtered out, and the spliced reads are retain; 2) the end of each candidate read are mapped to the genome to find unique anchor position; 3)candidate circRNAs are confirmed when the 3 'end of the anchor sequence is aligned to the upstream of the 5 'end of the anchor sequence, and the presumed breakpoint is flanked by GU/AG splicing signals. CIRI was first analyzed the CIGAR value in the sam file, scans the PCC signal (paired chiastic clipping signals) from the sam file, then filters the Junction reads based on the PEM and GT-AG signals, and finally detects unbalanced junction reads based on the DM algorithm. Filter and prevent false positives caused by homologous gene similarity and repeated sequences. We will choose two software for joint analysis to improve the accuracy of circRNA identification.

Small RNA tags were mapped onto the assembled genome of mus_musculus_Ensembl_97 using Bowtie $[36,37]$. The mapped small RNA tags were screened in the miRBase 20.0 database to search for known miRNA. In addition, modified software mirdeep2 and srna-tools-cli were used to obtain the potential miRNA and draw the secondary structures [38]. The hairpin structure of miRNA precursors can be used to predict novel miRNA. We integrate miREvo [39] and mirdeep2 [38] to predict the novel miRNAs. Meanwhile, custom scripts were used to obtain the identified miRNA counts and base bias on the first position with certain length and on each position of all identified miRNA, respectively [40]. miRNA expression was estimated using TPM (transcript per million) Density distribution[41]. Differentially expressed miRNAs were identified using the threshold: P-value $<0.05$.

\section{Differential expression gene analysis (DEG analysis) and Enrichment Analysis}

The gene expression level of RNA-seq is usually measured with FPKM (Fragments Per Kilobase of transcript sequence per Millions base pairs sequenced) [42]. We used a cutoff value of FPKM $>1$ to define the gene expression. Differential expression gene analysis was performed using edgeR [43]. We use $p$-value or corrected $p$ value (padj) to determine 
the significance level. The significant difference standard is padj less than 0.05. Differentially expressed genes are screened and subjected to hierarchical cluster analysis. GO (Gene Ontology) is a comprehensive database describing gene functions, which can be divided into three parts: molecular function, biological process, and cellular component. GO analysis was performed by GOseq [44] (Bioconductor, USA). The p-value of GO term and the false discovery rate (FDR) of p-value (q-value) were calculated to find out the most relevant GO term for the genes with differential expression. GO enrichment takes padj less than 0.05 (default) or pvalue less than 0.05 as significant enrichment. Pathway enrichment analysis was performed using KEGG database [45] (Kanehisa Laboratories, Japan). Hypergeometric test was applied to identify the most significant enriched pathways among the candidate differently expressed genes. Similarly, for KEGG pathway enrichment, pvalue or padj is less than 0.05 as significant enrichment.

\section{Construction of the ceRNA regulatory network}

The lncRNA-miRNA, miRNA-mRNA, lncRNAmiRNA-mRNA, circRNA-miRNA, miRNA-mRNA, and circRNA-miRNA-mRNA networks were developed based on possible functional relationships between DE-lncRNAs, DE-miRNAs, DE-circRNAs, and DE-mRNAs. The IncRNA-miRNA-mRNA ceRNA regulation network was based on the theory that lncRNAs can directly interact by invoking miRNA sponges to regulate mRNA activity [46]. "GDCRNA Tools" (http://bioconductor.org/packages/devel/ bioc/html/GDCRNATools.html) package in $\mathrm{R}$ software were used [47]. We use Cytoscape 3.6.1 software to plot lncRNA-miRNA-mRNA network (Biological network exploration with Cytoscape 3). The candidate miRNA-circRNA regulation network was predicted using miRanda (MicroRNA targets in Drosophila). Similarly, the target mRNAs of DEmiRNAs were predicted by scanning for conserved miRNA target sites with MiRanda (MicroRNA targets in Drosophila), and a miRNA-mRNA regulation network was constructed. We use Cytoscape 3.6.1 software to combine circRNA-miRNA network and miRNA-mRNA network to generate circRNAmiRNA-mRNA network (Biological network exploration with Cytoscape 3).

\section{Validation of the Differential Expression of InCRNA and circRNA}

To order to verify the results of transcriptome sequencing, we performed real-time PCR to determine the RNA levels of five lncRNAs and three circRNAs in chondrocyte and cartilage tissue. Quantitative real-time PCR amplification was performed by the ABI 7500 Real-Time PCR Systems (Applied Biosystems, USA). The first-strand cDNA was synthesized with PrimeScript ${ }^{\mathrm{TM}}$ RT Master Mix (Takara, Japan) and $2 \mu \mathrm{g}$ total RNA. The amplification procedure was one cycle of $30 \mathrm{~s}$ at $95^{\circ} \mathrm{C}$ for pre-degeneration followed by 40 cycles of 30 s at $95^{\circ} \mathrm{C}$ and $34 \mathrm{~s}$ at $60^{\circ} \mathrm{C}$. The $\mathrm{Ct}$ values of the reference gene and the target genes were obtained according to the amplification curve. The relative quantification of gene expression was obtained by using the $2^{-\Delta \Delta \mathrm{Ct}}$ method. GAPDH was used to normalize mRNA levels. The primer sequences used for real-time PCR are shown in Table 1.

Table 1. The IncRNA and circRNA number and their amplification primer.

\begin{tabular}{|c|c|c|c|}
\hline order name & Sequence & Length (nt) & Target- ncRNAs \\
\hline qmlncR4242F & TTCTCGGTGTCCAGGTTGC & 19 & >ENSMUST00000124242 \\
\hline qmlncR4242R & GCGGTAGTGAGTCGGTTTG & 19 & \\
\hline qmlncR4452F & TGCGTCCCCACTGACTAGC & 19 & >ENSMUST00000224452 \\
\hline qmlncR4452R & GTTGGCATCTGGACCATCTTC & 21 & \\
\hline qmlncR6685F & AATAAGGCAGCGGAAAGAAG & 20 & >ENSMUST00000126685 \\
\hline qmlncR6685R & AAGGCTCCCAATGACACG & 18 & \\
\hline qmlncR9207F & ACAGTGAGTCCTCCTTACAAAC & 22 & >ENSMUST00000169207 \\
\hline qmlncR9207R & ATGGCCTGGAACTTCTGTG & 19 & \\
\hline qmlncR9808F & САСССТСААТАСССТСАССТС & 21 & > ENSMUST00000219808 \\
\hline qmlncR9808R & AGCGGCAGTCTGTCTCATAC & 20 & \\
\hline qmcirR0172F & AGTCCGTCGTGTCGTTCCA & 19 & >mmu_circ_0000172 \\
\hline qmcirR0172R & CTGCTGGGTATAAATGGTAGGAG & 23 & \\
\hline qmcirR0571F & CCATCACGGGAGATTTTGC & 19 & >mmu_circ_0000571 \\
\hline qmcirR0571R & CACCAGAAAGGAACTGTCGC & 20 & \\
\hline qmcirR1775F & CAACСССТСТGACСТCTACG & 20 & >mmu_circ_0001775 \\
\hline qmcirR1775R & TCAGGCCAATGATGAAAAGC & 20 & \\
\hline qmcirR0710F & ACTGGCAGCACCACTAACAC & 20 & >mmu_circ_0000710 \\
\hline qmcirR0710R & CGAACTGCTCACTAAGGACG & 20 & \\
\hline
\end{tabular}




\section{Results}

\section{Identification of DE mRNAs between XBP I CKO mice and control mice}

To identify the expression levels of mRNAs in XBP1 CKO mice and control mice, 6 mRNA libraries were constructed. Compared with the wildtype control, 477 genes, including 307 (74.2\%) upregulated genes and 107 (25.8\%) downregulated genes, were differentially expressed in XBP1 CKO mice, with a q value $<0.05$ (Fig. $1 \mathrm{~A}$ and Table S1). Based on hierarchical cluster analysis, some of the 477 DEGs may participate in the regulation of the same pathway or possess similar functions (Fig. 1B and Table S2). Among these genes, genes related to cartilage development (derived from the GO terms GO:1990079, GO:0061037, GO:0060351, GO:0003417, GO:0061035, and GO:0051216) were significantly enriched after XBP1 knockdown. There were 5 out of 138 such genes, compared with 477 out of 55573 genes $(p=0.001$, hypergeometric test). To further explore the functions of these DEGs, GO and KEGG functional analyses were performed. GO is an international standard classification system for gene function. GO can be divided into three parts: biological process (BP), cellular component (CC), and molecular function (MF). A total of 274 significantly enriched GO terms were identified (Fig. 1C and Table S3), with $74.5 \%, 15.3 \%$, and $10.2 \%$ mRNAs assigned to BP, CC and MF, respectively. The MF category is associated with binding, protein binding and cytoskeletal protein binding; the BP category is associated with muscle structure development, muscle cell differentiation, muscle tissue development, and striated muscle cell differentiation; and the CC category is associated with contractile cytoplasm, cytoplasmic part, myofibril, sarcomere, contractile fiber and contractile fiber part. The DEGs were also aligned in the KEGG pathway database. As shown in Fig. 1D and Table S4, the pathways of PPAR signaling, hypertrophic cardiomyopathy (HCM), dilated cardiomyopathy (DCM), arrhymogenic right ventricular cardiomyopathy (ARVC) and calcium signaling were mainly activated.
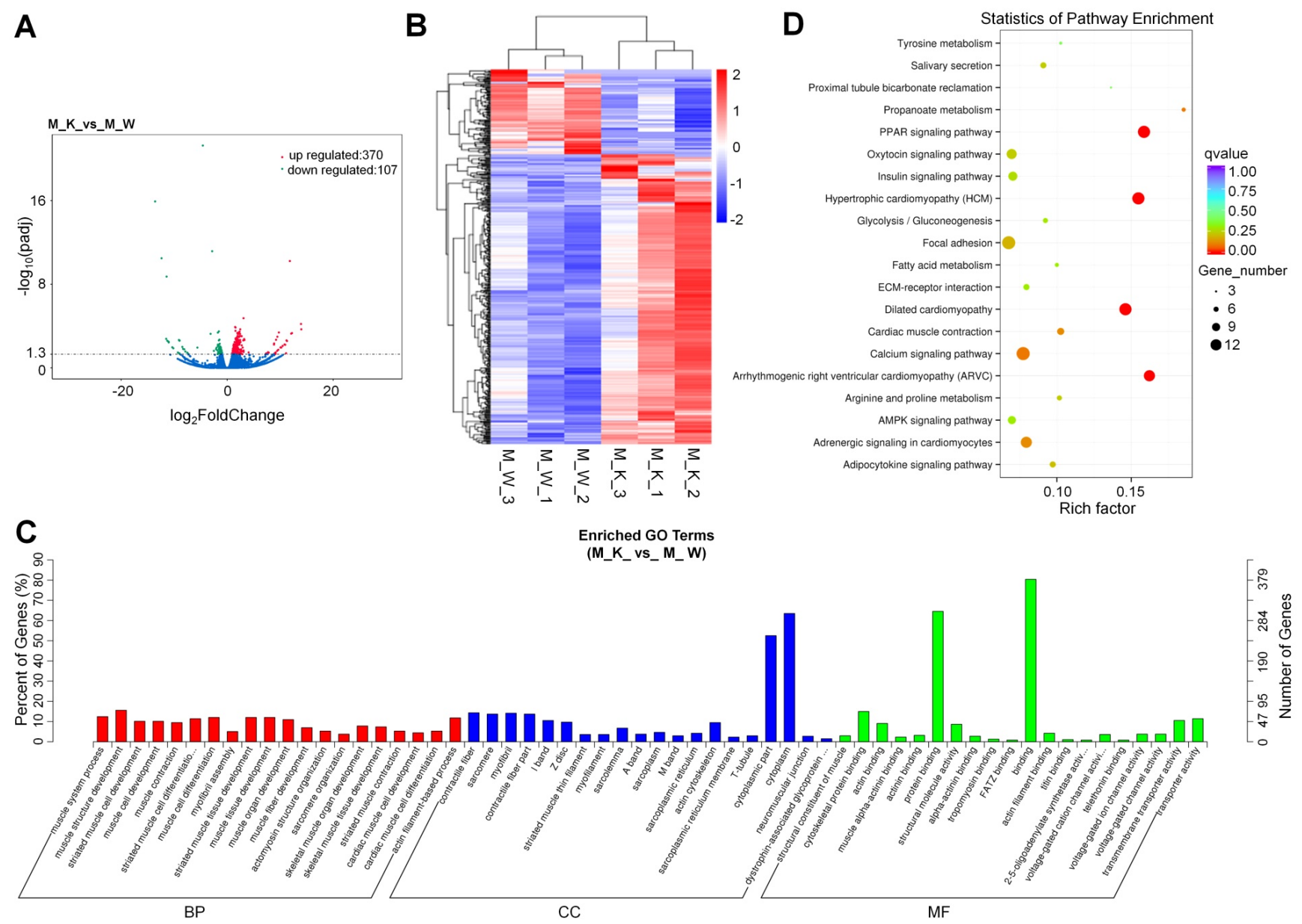

Figure 1. Overview of mRNA expression. (A) Volcano plots for mRNA. (B) Heatmap is used to assess the expression of mRNA. Red and blue denote high and low expression, respectively. Each DE-miRNA is represented by a single row of colored boxes, and each sample is represented by a single column. (C) Go term analysis of mRNA. (D) KEGG analysis of DE mRNA. 


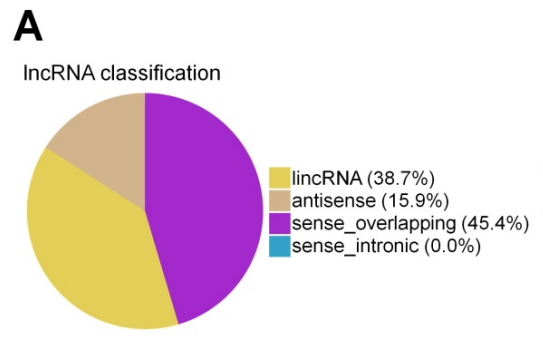

C

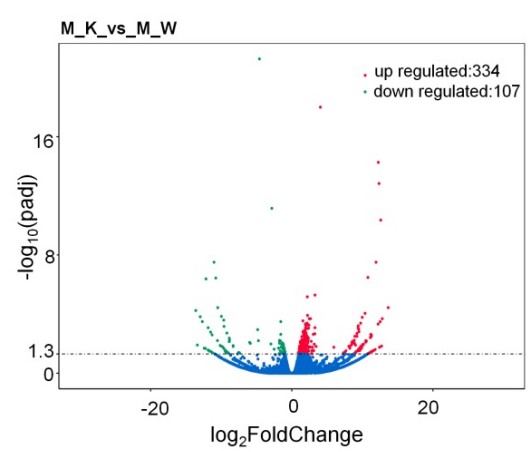

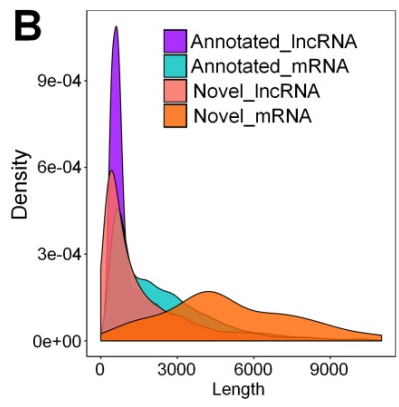

D

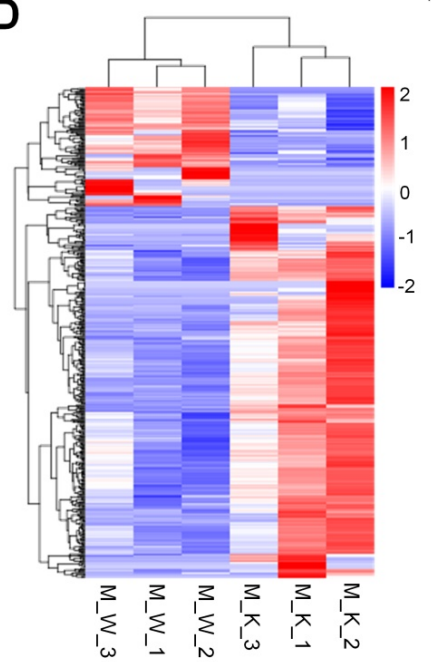

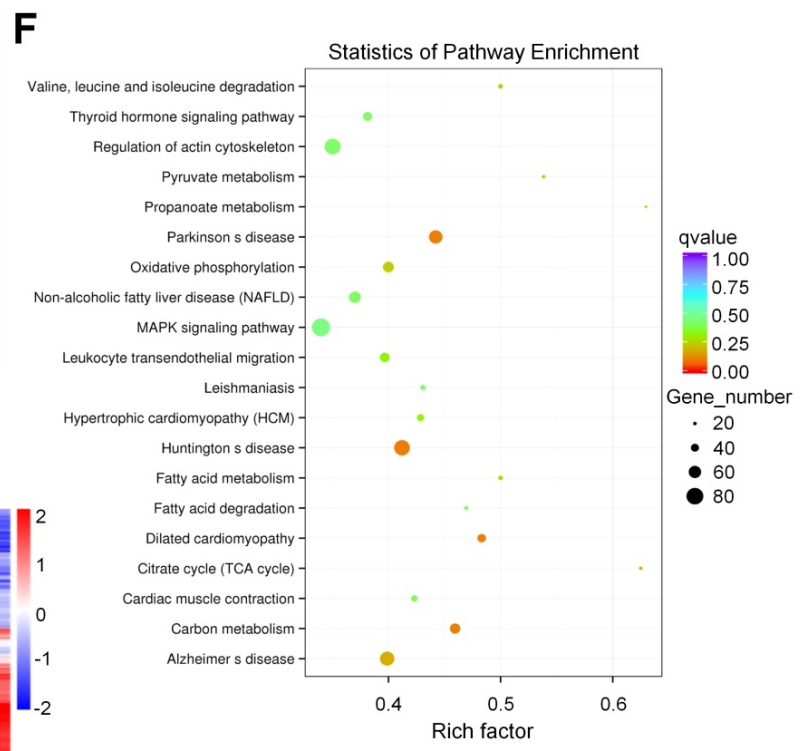

$\mathbf{E}$ Enriched GO Terms
(M_K_ vs_ M_W)

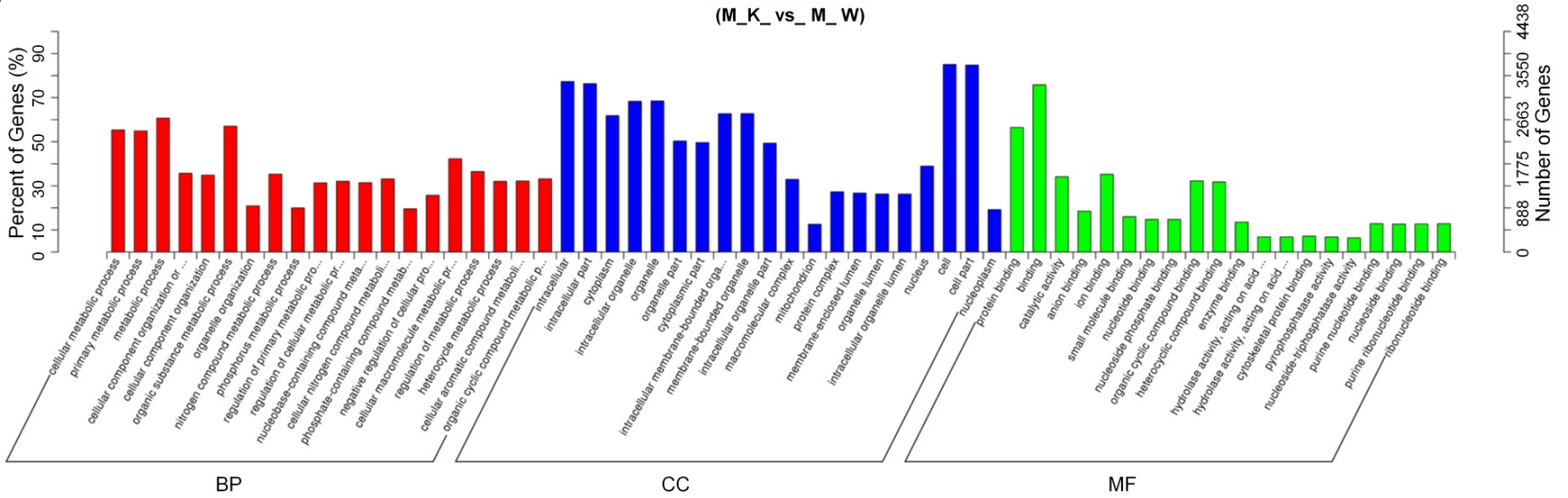

Figure 2. Prediction and function analysis of IncRNA target genes. (A) LncRNA type distribution. (B) Comparison of the length of IncRNA and mRNA density distribution. (C) Volcano plots for IncRNA. (D) Heatmap is used to assess the expression of IncRNA. (E) GO analysis of target genes of co-expression of differential IncRNA. (F) KEGG analysis of target genes of co-expression of differential IncRNA.

\section{Prediction and function analysis of the IncRNA target genes}

With reference to HUGO Gene Nomenclature Committee (HGNC), the newly screened lncRNAs were divided into four types according to their positional relationship with known mRNAs (Fig. 2A). To verify whether the new lncRNA meets the general characteristics, we compared the length of the transcript, the number of exons, and the length of the ORF between the lncRNA and the mRNA (Fig. 2B and Fig. S1A and S1B). Based on the figure, the new lncRNA conforms with the general characteristics of the transcript (transcripts with a transcript length longer than $200 \mathrm{nt}$ and number of exons greater than or equal to 2 (Fig. 2B and Fig. S1A and S1B)). The DEGs in the lncRNA expression patterns between XBP1 knockout mice and controls were analyzed, including 334(75.7\%) up-regulated genes and 107(24.3\%) down-regulated genes (Fig. 2C and Table S5). To explore the expression patterns of these lncRNAs, a hierarchical cluster analysis of 441 differentially expressed lncRNAs was performed between XBP1 knockout mice and controls, which classified the expression patterns of the XBP1 knockout mice and wildtype mice into different 
clusters (Fig. 2D and Table S6). We carried out target gene prediction for all lncRNAs, (i.e., predict the target genes of lncRNA through the co-location and co-expression of lncRNA and protein-coding genes). Thereafter, function enrichment analysis (GO/KEGG) of the target genes of the differential lncRNA was carried out to predict the main function of lncRNA. The prediction results of co-expression and co-location are shown in Fig. 2E, Fig. S2 and Tables S7 and S8). The 841 core GO terms and 45(co-location) were extracted (Fig. 2E, Fig S2, Tables S7 and S8). In the BP category, GO terms, such as cellular metabolic process, primary metabolic process, metabolic process, organic substance metabolic process, cellular macromolecule metabolic and nitrogen compound metabolic process, were functionally enriched. The CC category was found to be associated with cell, cell part, intracellular part, intracellular, organelle, intracellular organelle and cytoplasm which the MF category was found to be associated with binding, protein binding, ion binding, catalytic activity, organic cyclic compound binding and heterocyclic compound binding. To further focus on the function of these lncRNAs, the KEGG pathways were analyzed. Based on the results, the differentially expressed lncRNA is involved in 4(co-expression) (Fig. 2F and Table S9) and 4(co-location) (Fig. S3 and Table S10). KEGG metabolic pathways, such as Parkinson's disease (PD), Huntington's disease (HD), carbon metabolism and dilated cardiomyopathy (DCM), were identified (Fig. 2F). The prediction result of co-location is shown in Fig. S4 and Table S10.

\section{Overview of the circRNA sequencing data}

To understand the roles of XBP1-associated circRNAs in cartilage development, we performed circRNA sequencing using the rRNA-depleted chondrocyte samples of wildtype mice $(\mathrm{CON})$ and XBP1 CKO mice. Clean reads from the 6 libraries were used for circRNA identification. After a series of selection, 1651 novel circRNAs, which were widely distributed on 21 chromosomes were obtained and termed as mmu_circ_0000004 to novel_circ_0003429 (Fig. 3A, Fig. S4 and Table S11). A size distribution analysis revealed that the length of most circRNAs ranged from 200 to $400 \mathrm{bp}$ (Fig. 3B). By counting the sources of circRNA in all samples, we found that $95.08 \%$ of circRNAs were composed of exons, while $3.34 \%$ and $1.58 \%$ were located in the intronic and intergenic regions, respectively (Fig. 3C). To identify circRNAs that potentially participated in cartilage development, the differences in circRNA expression patterns between XBP1 CKO mice and wildtype mice were analyzed. As shown in Table S12, a total of 15 differentially expressed circRNAs were observed relative to the control, including 10 upregulated genes and 5 downregulated genes (Fig. 3D). To explore the expression patterns of these circRNAs, a hierarchical cluster analysis of 15 differentially expressed circRNAs was performed between the XBP1 CKO mice and wildtype mice, which classified the expression patterns of samples from XBP1 CKO mice and wildtype mice into different clusters (Fig. 3E). GO and KEGG functional analyses of these circRNAs were also conducted to explore the biological function of circRNAs. GO analysis, revealed that the regulation of cell cycle process, positive regulation of cytokinesis and cell division, regulation of cytokinesis, cell cycle arrest, cytokinesis and maintenance of location in cell were enriched in the BP category. The dominant functions in each of the remaining two categories included cytoplasm and cytoplasmic part in the CC category and purine ribonucleoside triphosphate and enzyme regulator activity in the MF category (Fig. 3F and Table S13). KEGG pathways analysis, which revealed the function of these circRNAs, showed that the pathways of cell cycle, and metabolic pathway were activated (Fig. 3G and Table S14).

\section{Overview of the miRNA sequencing data}

We identified miRNAs in XBP1 CKO mice and explored their expression patterns. We found that $90.01 \%$ to $91.27 \%$ of small RNA reads were mapped onto the mouse (mus_musculus_Ensembl_97) genome (Table S15). The length distribution of sRNA was counted, and was found to range from 18 to $35 \mathrm{nt}$, with a peak of $22 \mathrm{nt}$, followed by $21 \mathrm{nt}$ and $23 \mathrm{nt}$, respectively (Fig. 4A and Fig. S5). The statistics and annotations of all small RNAs and various types of RNAs are summarized in Fig. 4B and S6 Fig. We proceeded to predict the new miRNA. M_K_1 as an example, a total of $12,993,994$ miRNAs were identified, including 6,126,475 known miRNAs and 830 novel miRNAs (Fig. 4B). The predicted new miRNA and the comparison of each sample sRNA are presented in Table S16. The expression levels of new and known miRNAs in each sample were determined, and the TPM (number of transcripts per million clean tags) density distribution was used to verify the gene expression pattern of the sample as a whole (Fig. 4C). The expression of miRNAs in different groups was compared, and the overall expression patterns of miRNAs in these groups were found to be highly consistent (Fig. 4C). Compared with the miRNA expression levels of the control group, 6 miRNAs showed significantly differential expression $(p<0.05)$, including 1 significantly up-regulated miRNA and 5 significantly down-regulated miRNA (Fig. 4D). To explore the expression patterns of these miRNAs, a hierarchical clustering analysis of 6 differentially 
expressed miRNAs was performed. Based on the results of this analysis, the cartilage samples of XBP1 $\mathrm{CKO}$ mice and control mice were divided into different clusters (Fig. 4E). After further evaluation, we found that these miRNAs target 43,617 transcripts. According to the correspondence between miRNAs and their targets, we performed GO and KEGG enrichment analysis on each set of differentially expressed miRNA target genes. GO analysis showed that these target genes were mainly enriched in 468 GO term processes $(p<0.05)$ (Fig. 4F). Analysis of the KEGG pathways revealed that these miRNA target genes were involved in the cancer pathway, Hepatitis $\mathrm{B}$ and caffeine metabolism, thyroid hormone signaling pathway, and mucin type O-glycan biosynthesis (Fig. 4G).
A

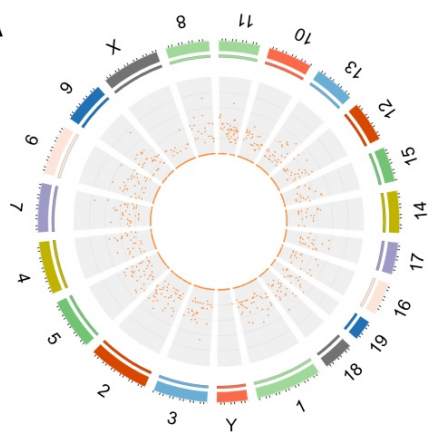

D

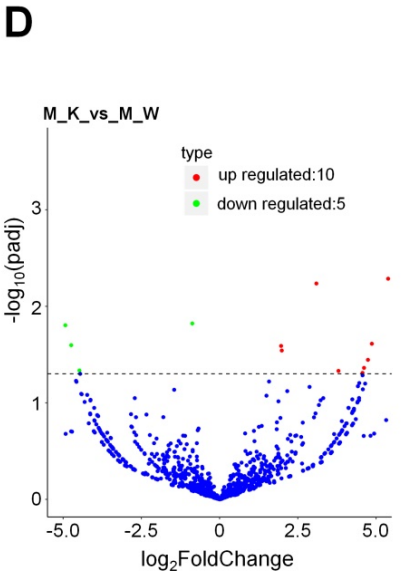

E
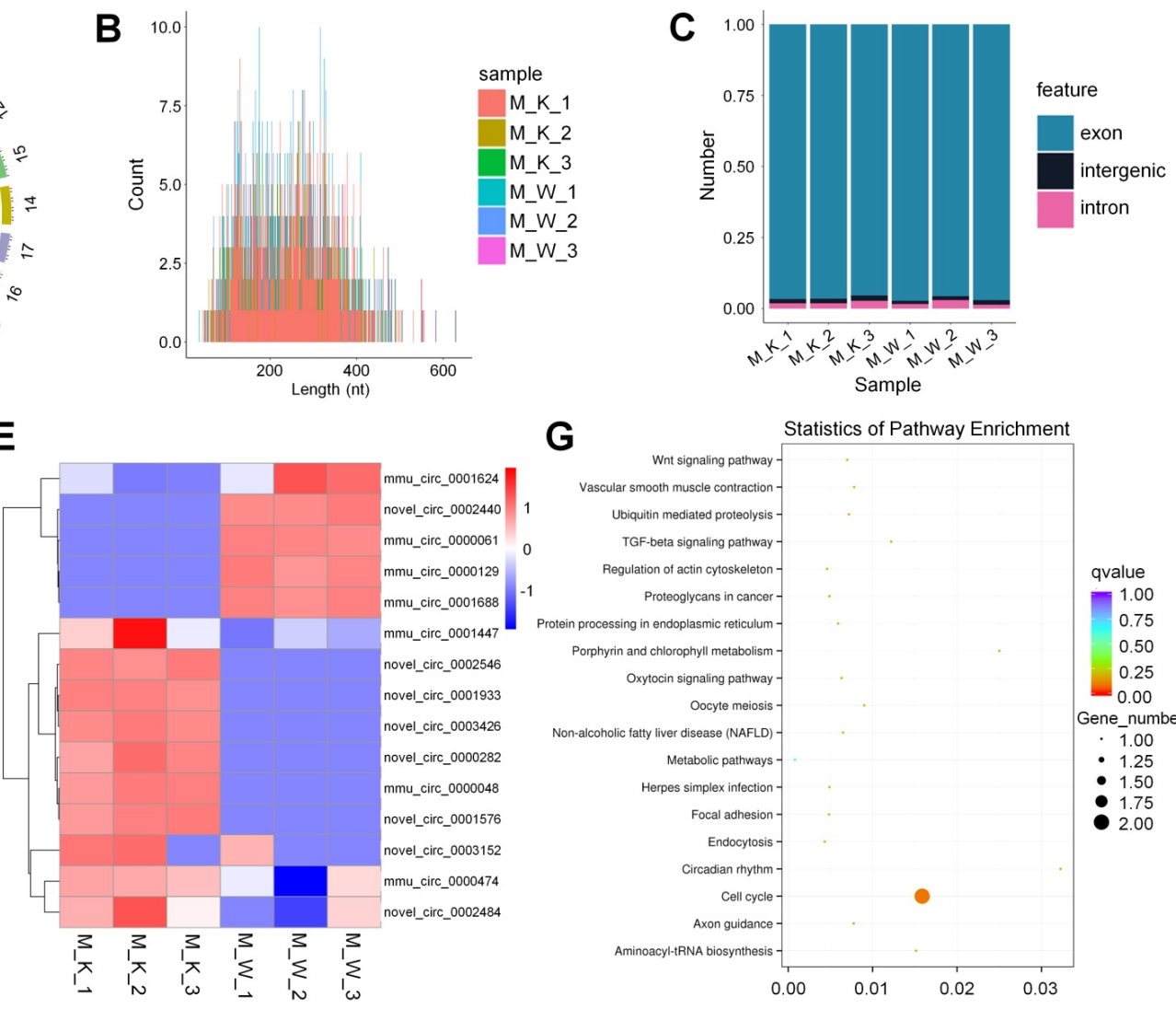

$G$
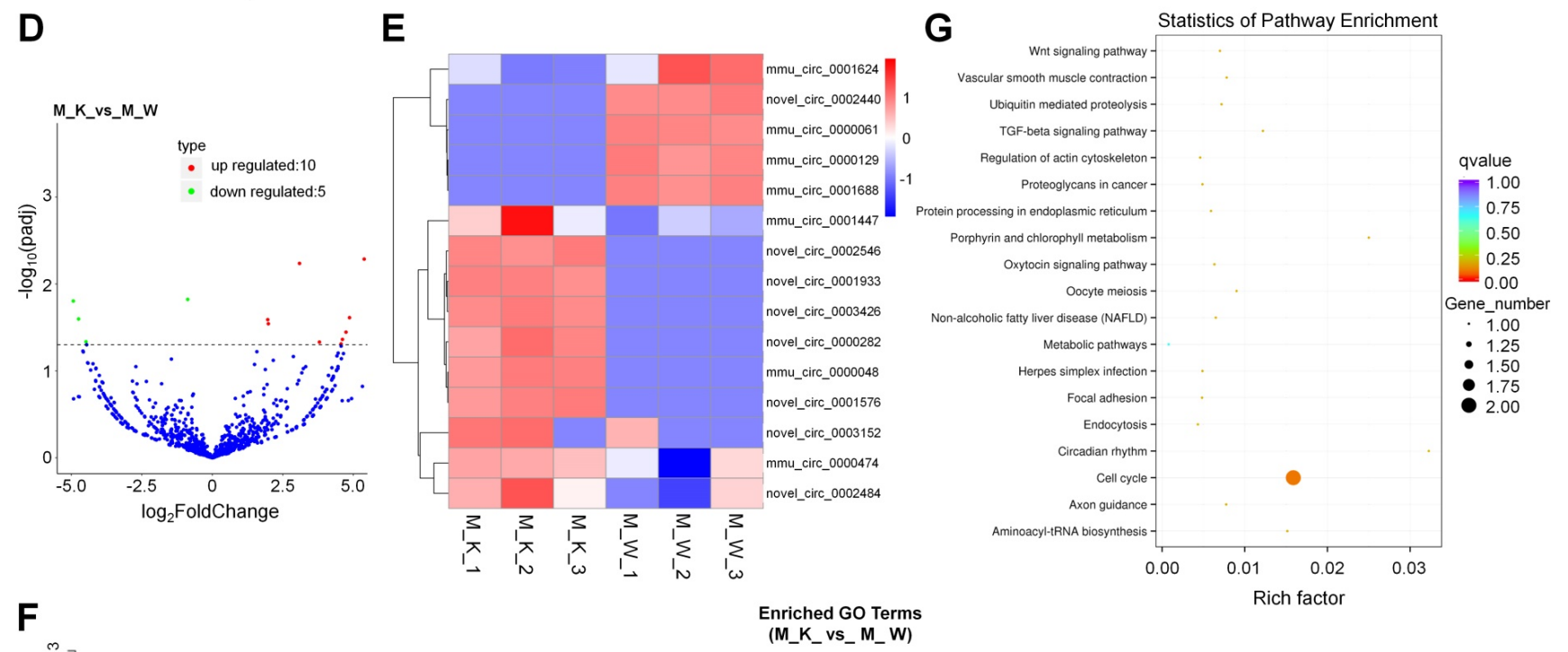

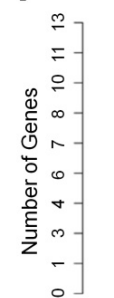

Enriched GO Term 
A

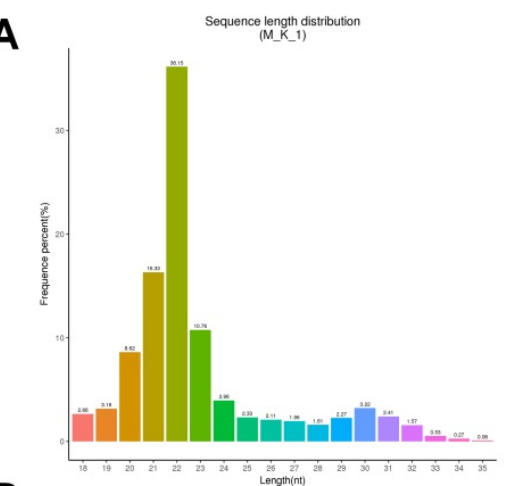

D

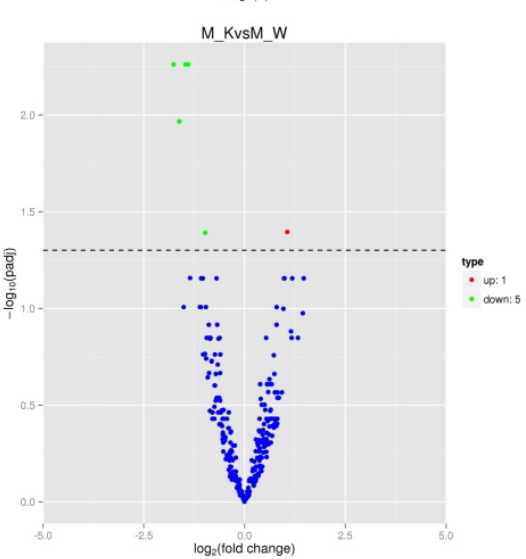

$\mathbf{F}$

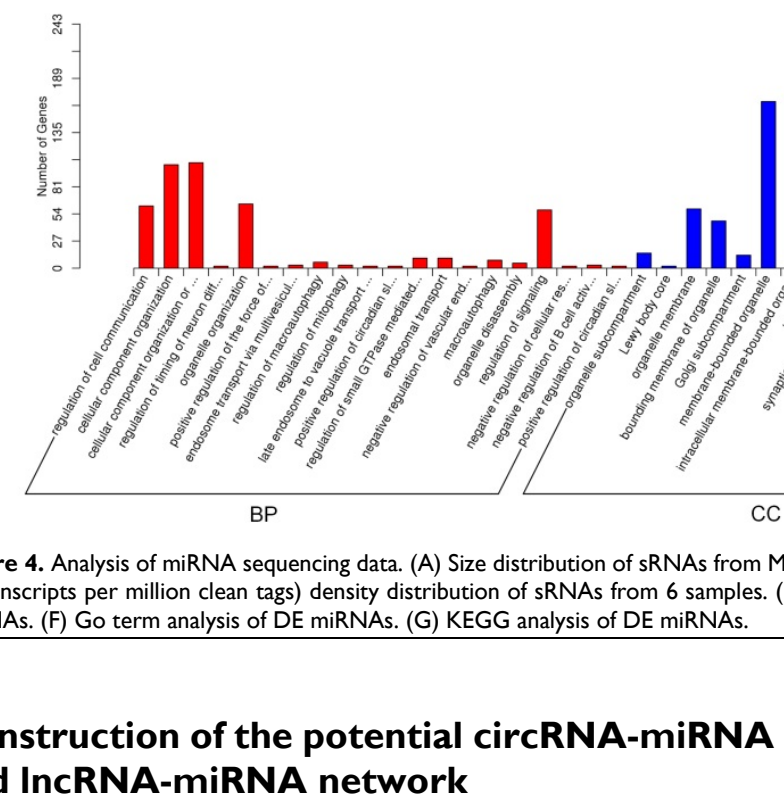

Competitive endogenous RNA (ceRNA) analysis is directly based on the ceRNA hypothesis, centered on miRNA, and analyzed based on the correlation between the expression levels of different molecules in multiple samples. Further, this assessment does not rely on difference analysis [48]. To clarify the functional relationship between lncRNA and its target miRNA, and circRNA and its target miRNA, we conducted a comprehensive analysis of the interaction between lncRNA and its target miRNA, and circRNA and its target miRNA. According to the expression values of miRNA and circRNA, 5,882 miRNA-
circRNA interactions were identified (absolute correlation coefficient $>0.7$ and $p$-value $<0.05$ ), with 974 miRNAs and 1,627 circRNAs, respectively (Table S17). Among these interactions, 5,055 were determined to be positively correlated, while the remaining 827 were negative (Table S17). Similarly, 272,329 miRNA-lncRNA interactions were identified (absolute correlation coefficient $>0.7$ and p-value $<0.05$ ), including 1,076 miRNA and 37,320 lncRNAs, respectively (Table S18). Among these interactions, 178473 were determined to be positively correlated, while the remaining 93,856 were negative (Table S18). Further, we found that 14 circRNAs (mmu_circ 0001376, mmu_circ_0001632, mmu_circ_0000713, mmu_circ_0000714, novel_circ_0002622, novel_circ_ 
0002301， novel_circ_0002887, novel_circ_0002720, novel_circ_0000181, novel_circ_0000182, novel_circ_ 0000199, novel_circ_0000280, novel_circ_0000108 and novel_circ_0000140) had binding sites for mmu-miR99b-5p, which targets ENSMUSG00000031849 (log2 fold change $=4.59) \quad($ Table S19). LncRNAs (ENSMUST00000206990 ( $\log 2$ fold change $=0.98)$ ) had binding sites for mmu-miR-23a-5p, which targets ENSMUSG00000000126 (log2 fold change=3.19) (Table S20). In summary, a deficiency in XBP1 in chondrocyte leads to changes in the expression of some miRNAs, lncRNAs and circRNAs, which are positively or negatively correlated with target marker genes that regulate cartilage growth. $\mathrm{XBP} 1$ may thus participate in the process of bone and cartilage growth through the network of miRNAs-lncRNA or miRNAs-circRNAs, which play an important role in cartilage growth and bone associated diseases.

\section{Construction of the potential miRNA-mRNA network}

We used the miRNA software to construct a regulatory network of miRNA and its corresponding target mRNA. Collectively, we identified 14141 miRNA-mRNA interactions, involving 907 and 8758 miRNA and mRNAs, respectively (Table S21). Most of these miRNAs were found to target multiple mRNAs. For example, mmu-miR-709, mmu-miR-149-3p, mmu-miR-1249-5p, mmu-miR-7648-3p and mmumiR-328-5p were found to have 177, 195, 166, 174, and 68 target mRNAs, respectively. Further, 136 types $(14.99 \%)$ of miRNAs were found to only target one mRNA. Many mRNAs were related to more than one miRNA. For example, ENSMUST00000108883 was targeted by 2 miRNAs, including mmu-miR-3154 and mmu-miR-370-5p. Such findings indicate a complex miRNA-mRNA regulatory network in the process of cartilage development, the involvement of some miRNAs in cartilage development through the directly targeting of the mRNA of some cartilage growth related marker genes. Other miRNAs were found to participate in cartilage development through the network of miRNA-lncRNA or miRNA-circRNA.

\section{Comprehensive analysis of the circRNA--miRNA-mRNA and IncRNA-miRNA-mRNA networks}

To further explore the potential networks among circRNA, miRNA, and mRNA, we combined the circRNA-miRNA interaction and miRNA-mRNA interaction to construct 1,822 circRNA-miRNAmRNA interactions (satisfying $|R|>0.7$ and $p<0.05$ for both circ-mRNA and miRNA-mRNA interactions) (Fig. 5C), that involve 203 circRNAs, 186 miRNAs, and 893 mRNAs (Table S22). The circRNA-miRNA-
mRNA networks suggested that 11 upregulated circRNAs were bound to 7 miRNAs and 34 miRNA-targeted mRNAs. Three down-regulated circRNAs (novel_circ_0001966, novel_circ_0002499, and mmu_circ_0000906) harbored the miRNA mmu-miR-3102-3p which targeted the BIG-2A gene and may be related to the down regulation of that gene. Meanwhile, the upregulated circRNAs mmu circRNA, mmu_circ_0000905, and mmu_circ_0000950, harboring Anx6 target the miRNA mmu-miR-3153 and mmu-miR-370-5p, which may be related to its up regulation.

Based on our prior results, 204 differentially expressed lncRNAs, 530 differentially expressed mRNAs, and 1076 differential expressed miRNAs that might play a role in cartilage development were revealed. We constructed 253235 lncRNA-miRNAmRNA co-expression networks based on the lncRNA-miRNA and miRNA-mRNA results (satisfying $|R|>0.7$ and $p<0.05$ for both circ-mRNA and miRNA-mRNA interactions) (Fig. 5F and Table S23). A remarkable number of lncRNA was determined to be candidate inhibitors for the repressive effect of miRNAs to their targets. For example, there were 311 lncRNAs including highly upregulated $\mathrm{HO} 2$, harboring the targeting miRNA of the Vgrl gene and may be related to its induction of expression after XBP1 knockout. Similarly, another 31 downregulated lncRNAs harboring the targeting miRNAs of the Prhr1 and may be related to its increased expression level.

The networks between circRNA, lncRNA, miRNA and mRNA are complicated. In fact, the same miRNA can target different cirRNAs, lncRNAs and mRNAs, and different miRNAs can target different cirRNAs, lncRNAs and same mRNAs, Different circRNA-lncRNA -miRNA-mRNA network perform different biological functions according to the non-coding RNAs involved, which can be used as candidates for subsequent functional analysis.

\section{GO and KEGG analysis of IncRNA and circRNA regulatory networks}

We performed GO and KEGG analyses to evaluate the function of DEGs in the network. In the circRNA regulatory networks, GO analysis revealed that there were 119, 24, and 4 enriched GO terms with statistical significance $(p<0.05)$ in the BP, CC, and MF, respectively (Fig. 5A and Table S24). For BPs, the DEGs were enriched in cellular component organization, negative regulation of biological process, location and macromolecule modification. For CC, the top enriched items were intracellular, intracellular organelle, cytoplasm and membranebounded organelle. 
A

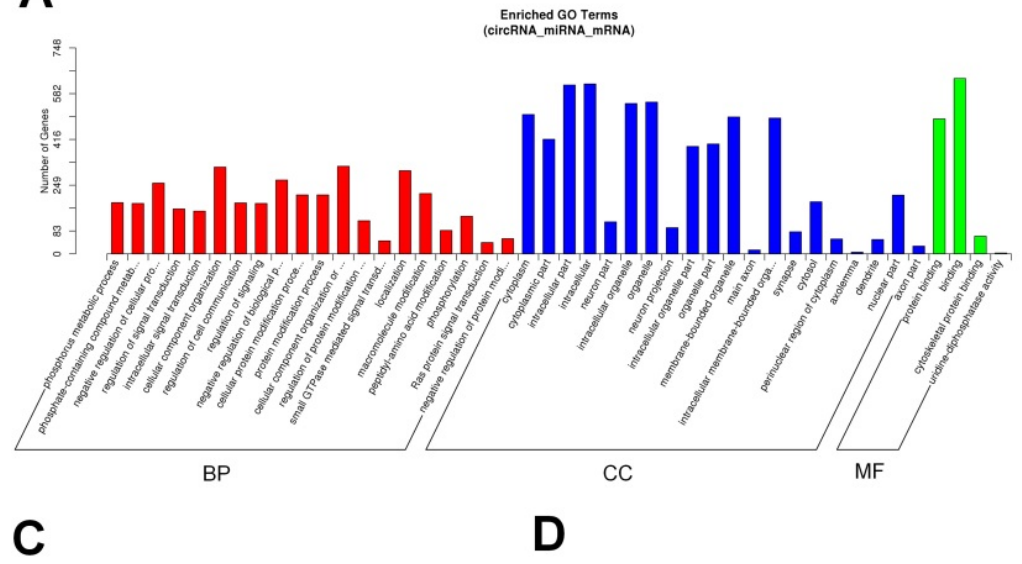

B

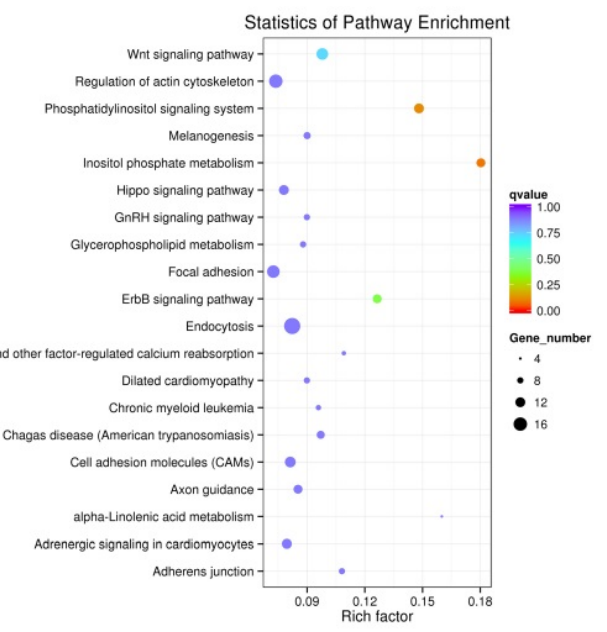

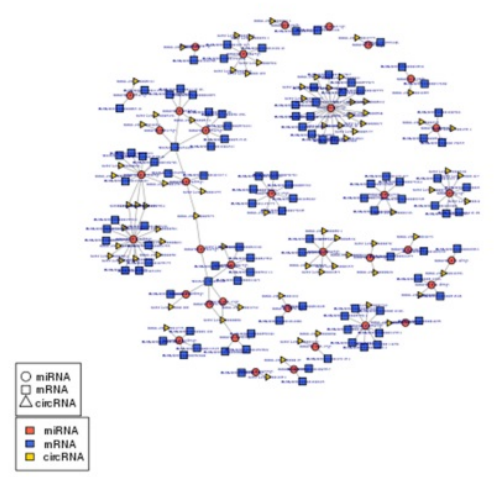

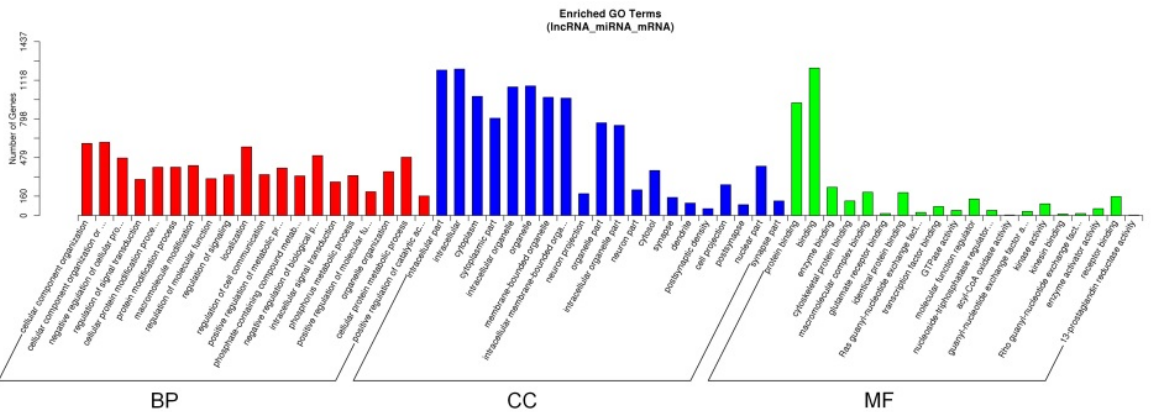

$\mathbf{E}$

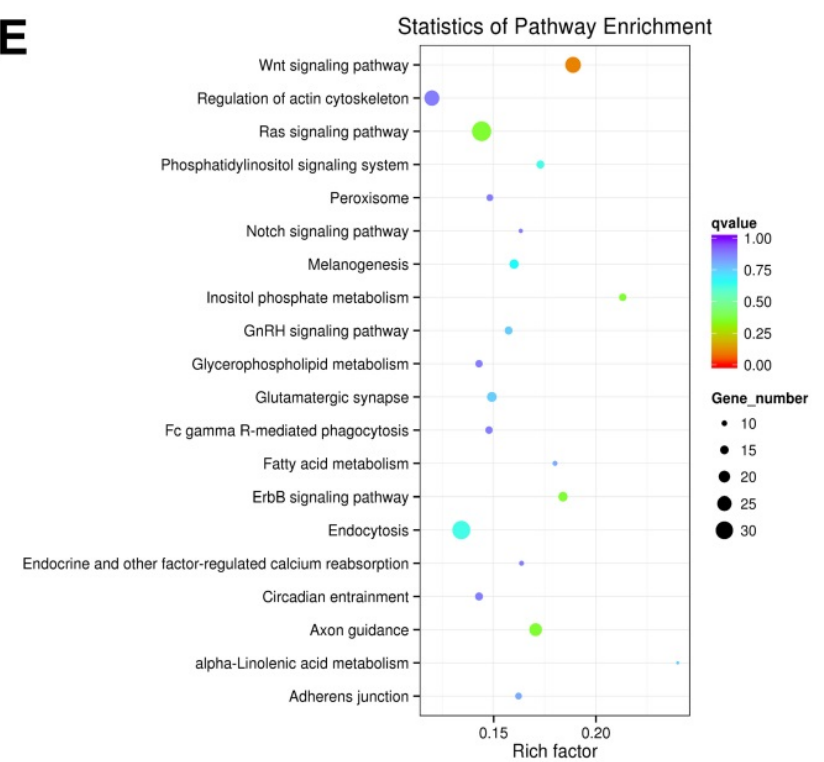

$\mathbf{F}$
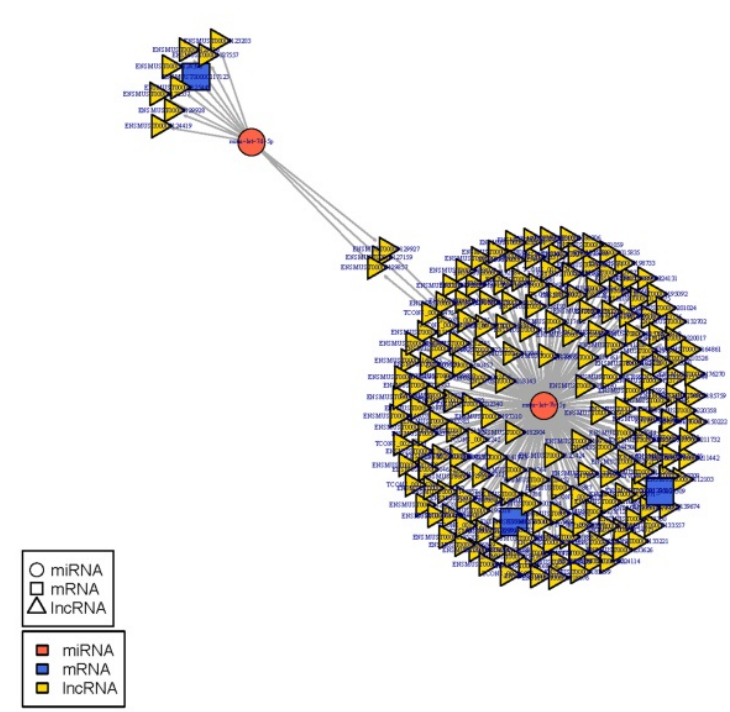

Figure 5. Established circRNA-miRNA-mRNA and IncRNA-miRNA-mRNA network.(A)GO analysis of the DEGs in the circRNA-miRNA-mRNA network. (B)KEGG analysis of the DEGs in the circRNA-miRNA-mRNA network. (C)The circRNA-miRNA-mRNA Competing endogenous RNA network. The squares indicate mRNAs in blue, triangles represent IncRNAs in light yellow and round shapes represent miRNAs in orange. (D)GO analysis of the DEGs in the IncRNA-miRNA-mRNA network. (E) KEGG analysis of the DEGs in the IncRNA-miRNA-mRNA network. (F)The IncRNA-miRNA-mRNA Competing endogenous RNA network. The squares indicate mRNAs in blue, triangles represent IncRNAs in light yellow and round shapes represent miRNAs in orange. 
A B

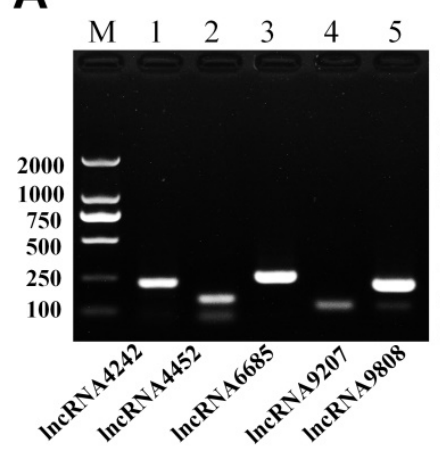

E

The cyclization site of mmu_circ_0000172

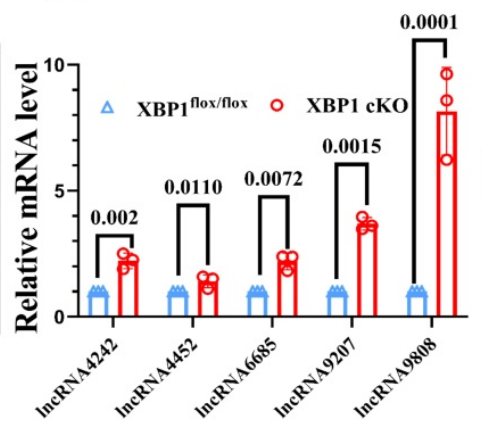

C

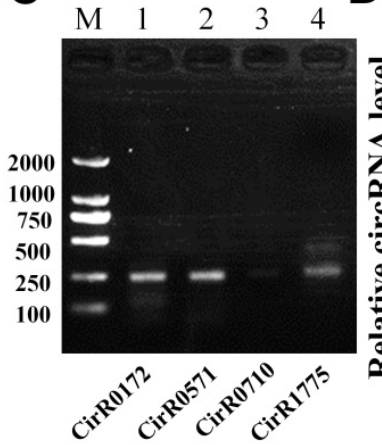

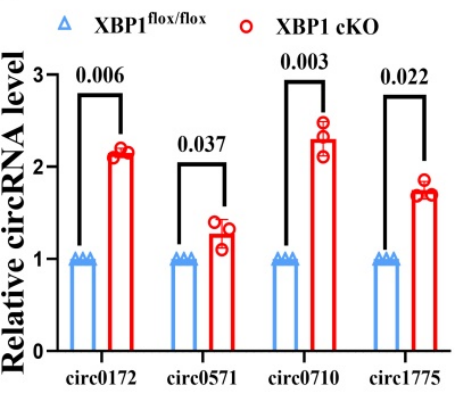

G

$\mathbf{F}$

The cyclization site of mmu_circ_0000571

The cyclization site mmu_circ 0001775

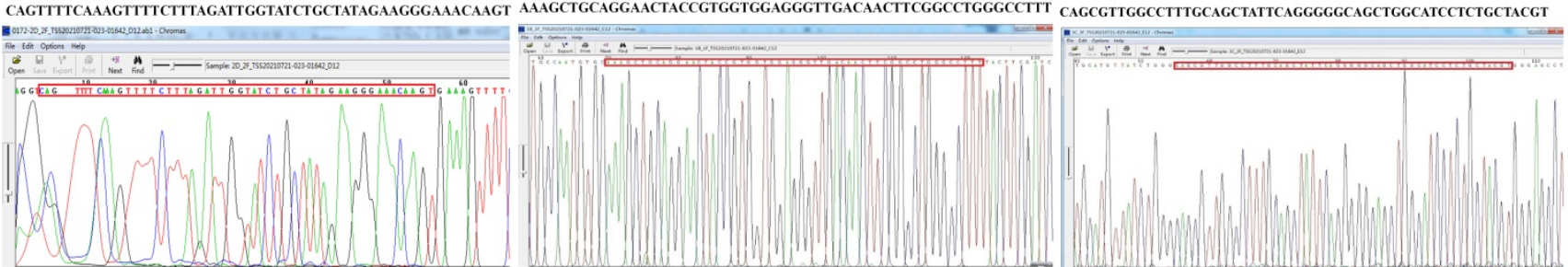

Figure 6. Confirmation the differential expression of ncRNAs in XBP1CKO and control mice. (A) The PCR detection of five IncRNAs. M. Marker, 1. ENSMUST00000124242, 2. ENSMUST00000224452, 3. ENSMUST00000126685, 4. ENSMUST00000169207, 5. ENSMUST00000219808. (B) mRNA level of five IncRNAs in the cartilage of XBPI CKO mice and control mice. (C) The PCR detection of four circRNAs. M. Marker, 1. mmu_circ_0000172, 2.mmu_circ_0000571, 3. novel_circ_0000710, 4.mmu_circ_0001775. (D) mRNA level of four circRNAs in the cartilage of XBPI CKO mice and control mice by QPCR. CircRNA sequencing of mmu_circ_0000172. (E) mmu_circ_0000571. (F) mmu_circ_0001775 (G).

Finally, for MF, binding and protein binding were enriched. KEGG pathway enrichment analysis was also conducted to characterize the target genes. Accordingly, the top significantly enriched pathways were identified to be related to inositol phosphate metabolism and phosphatidylinositol signaling system (Fig. 5B and Table S25). In the lncRNA co-expression networks, GO analysis revealed 298, 67, and 24 enriched GO terms with statistical significance $(\mathrm{p}<0.05)$ in the BP, CC, and MF, respectively (Fig. 5D and Table S26). The MF category had enriched terms in protein binding and binding. The BP category had enriched terms in intracellular component organization, localization, negative regulation of biological process and cellular protein metabolic process. The CC category had enriched terms in intracellular, intracellular organelle, membranebounded organelle and organelle part. KEGG pathway enrichment analysis indicated that the top significantly enriched pathway was Wnt signaling pathway (Fig. 5E and Table S27).

\section{Validation of the differentially expressed ncRNAs in mice}

To confirm the expression levels of RNA obtained by Illumina sequencing, we first selected five lncRNAs, including ENSMUST00000124242 (log2 fold change=3.06), ENSMUST00000224452 (log2 fold change=2.18), ENSMUST00000126685 (log2 fold change $=4.98), \quad$ ENSMUST00000169207 (log2 fold change=2.13), and ENSMUST00000219808 (log2 fold change $=5.78$ ), and measured their expression levels using RT-PCR (Fig. 6A). Thereafter, we isolated the cartilage tissue from XBP1 CKO (XBP1flox/flox col2CRE+) and control (XBP1flox/flox col2CRE-) mice, and detected the mRNA level of five lncRNAs in different cartilage tissues. QPCR revealed that the expression of five lncRNAs were upregulated in the cartilage of $\mathrm{XBP1} \mathrm{CKO}$ mice compared with that of controls (Fig. 6B).

We selected four circ-RNAs, including $\mathrm{mmu}_{-}$ circ_0000172 (log2 fold change=2.40), mmu_circ_ 0001775 ( $\log 2$ fold change=1.72), novel_circ_0000710 ( $\log 2$ fold change $=0.64)$, and mmu_circ_0000571 (log2 fold change $=0.70$ ), and detected their expression as well as their cyclization sites. The results of RT-PCR, qPCR and circRNA sequencing were consistent with those of Illumina sequencing. As depicted in Fig. $6 \mathrm{C}$, D, E, F, G, the cyclization sites were determined by circRNA sequencing, and the expression of three circRNAs was found to be upregulated in the cartilage of XBP1 CKO mice compared with that of controls. The primers sequences are listed in Table 1 . All of the randomly selected lncRNA and circRNA showed a similar expression pattern between $\mathrm{qPCR}$ and Illumina sequencing. Further, the both RT-PCR, qPCR and circRNA sequencing results confirmed the accuracy and reliability of the sequencing data. 


\section{Discussion}

Abnormal differentiation of chondrocytes will lead to several cartilage or bone developmental diseases, which are closely related to the molecular mechanism of their differentiation and development [49]. Previous studies have shown that the IRE1/XBP1s signaling pathway is involved in cartilage formation and bone growth [50]; however, the regulation mechanism of XBP1 and its associated non-coding RNA involved in cartilage development remain unclear. In the present study, we established $\mathrm{XBP1} \%$ cartilage-specific knockout mice (XBP1 CKO) and conducted whole transcriptome sequencing using cartilage tissue obtained from XBP1 CKO mice and controls. Whole transcriptome sequencing can reveal the target regulate relationship between various non-coding RNAs and mRNAs and the interaction network between ncRNAs. First, we analyzed differentially expressed circRNAs, IncRNAs, miRNAs, and mRNAs, which revealed $15 \mathrm{DE}$ circRNAs, 441 DE lncRNAs, 6 miRNAs and 477 mRNAs. Among them, genes related to cartilage development (derived from the GO terms GO: 1990079, GO: 0061037, GO: 0060351, GO: 0003417, GO: 0061035, and GO: 0051216) were significantly enriched after XBP1 knockdown. There were 5 out of 138 such genes, compared with 477 out of 55573 genes $(p=0.001$, hypergeometric test). The biological functions of the above RNAs were analyzed by GO and KEGG enrichment analysis.

With the development of molecular biotechnology, non-coding RNA, a type of RNA that cannot encode proteins, was considered to be noise in the genome in the past, and it plays a vital role in various biological processes [51]. Some studies found that the expression disorder of IncRNA or circRNA was related to the occurrence of bone development disease, such as lncRNA - OG [23] and circRNA_0001052/miR-124-3p [27]. However, whether and how XBP1 splicing regulates the formation and function of ncRNAs remain unknown. Further, whether and how XBP1 modulates cartilage development through these ncRNAs-mediated regulatory networks need to be clarified. Herein, some lncRNA-miRNA-mRNA and circRNA-miRNAmRNA networks related to XBP1 deficiency were constructed. These networks indicated that circRNA and lncRNA may play a nodal regulatory role. A single circRNA and lncRNA can be associated with multiple mRNAs and multiple identical miRNAs. For example, 14 circRNAs (mmu_circ_0001376, mmu_ circ_0001632, mmu_circ_0000713, mmu_circ_0000714, novel_circ_0002622, novel_circ_0002301, novel_circ_ 0002887, novel_circ_0002720, novel_circ_0000181, novel_circ_0000182, novel_circ_0000199, novel_circ_
0000280, novel_circ_0000108 and novel_circ_0000140) were found to have binding sites for mmu-miR99b-5p, which targets ENSMUSG00000031849 (log2 fold change $=4.59$ ) (Table S19). Hsa circ_0000729 was reported as a potential prognostic biomarker in lung adenocarcinoma, and hsa-miR-3154 serves as a potential prognostic biomarker for cervical cancer[52, 53]. MicroRNA is a type of small RNA molecule, that is usually combined with a short complementary sequence located in the $3^{\prime}$ _UTR region of mRNA to regulate the expression of target mRNA. MiRNA can directly regulate gene expression by interacting with not only lncRNA, but also circRNA and mRNA. Furthermore, both lncRNAs and circRNAs can regulate microRNAs through the "sponge" effect. LncRNAs, miRNAs, and circRNAs can also modulate gene expression by binding to the mRNA or mRNA splicing [12, 54-56].

LncRNAs are a highly heterogeneous class of RNAs that can be transcribed from any part of protein-coding genes and intergenic regions in sense or antisense orientation. The functions of lncRNA in eukaryotic cells are very diverse, including roles in high-order chromosome dynamics, telomere biology, and subcellular structure organization [10, 11, 57]. We found that lncRNAs (ENSMUST00000206990 (log2 fold change $=0.98)$ ) possessed binding sites for $\mathrm{mmu}-$ miR-23a-5p, which targets ENSMUSG00000000126 (log2 fold change=3.19) (Table S20). MiRNAs are thought to modulate the translation process of more than $60 \%$ of protein-coding genes in eukaryotic cells, which are involved in the regulation of many biological processes, including proliferation, apoptosis, differentiation, and development. Different miRNAs have different regulatory processes; some miRNAs control specific individual targets, whereas others serve as the main regulators of one process. Therefore, pivotal miRNAs can simultaneously regulate the expression levels of multiple genes, and different types of miRNAs coordinate their targets $[10,11,57]$. In XBP1 KO chondrocytes, mmu-miR-709, mmu-miR-149-3p, mmu-miR-1249-5p, mmu-miR7648-3p and mmu-miR-328-5p had 177, 195, 166, 174 and 68 target mRNAs, respectively. In summary, these RNAs play an important role in cartilage development by modulating XBP1 expression. In addition, the biological function of target genes was identified based on GO annotation and KEGG pathway enrichment analyses.

All is known that the coordinated regulation between different tissues and organs is performed by blood and body fluid circulation. The chondrocyte secretome is made up of many proteins participated in cellular regulatory pathways, cell-cell or cell-ECM interactions, including chaperons, apolipoproteins 
and chondrocalcin, etc. Chondrocyte metabolism is affected by its micro-environment, including various different secreted factors and ncRNAs. Furthermore, the chondrocyte micro-environment is also influenced by many kinds of secret proteins released from osteoblasts, osteocytes, osteoclasts [58-60]. XBP1 deficiency in chondrocyte causes differences in various of secreted factors and full transcriptome RNA, including all kinds of ncRNAs with differential expression and regulatory profiles as well as differences in protein expression and abnormalities of various different secreted factors. We analyzed the full profile of transcriptome RNAs, and found that XBP1 deficiency cartilage not only affects cartilage and related diseases influenced by the transcriptome, but also the functions of other tissues influenced by the transcriptome. Compared with those of normal mice, the expression and regulation profiles of not only multiple genes associated with many aspects of cartilage development and other cartilage-related diseases but also corresponding genes in other tissues and organs were altered in XBP1 CKO mice. Such changes result in different diseases in different tissues. As summarized in Fig. 7, differential ncRNAs and secreted factors caused by the lack of XBP1 in chondrocytes may cause abnormal signaling pathways in different organs and tissues through blood and body fluid circulation [60-62]. These differential signal pathways cause abnormalities in the functions of other tissues and organs, including the nervous system, heart, bone, thyroid, and intestine. Such abnormalities result in many types of diseases, including HCM, DCM, ARVC, PD, HD, and thyroid associated disease.

In summary, XBP1s is produced under UPR in ER stress. We analyzed the expression of ncRNAs and the network between circRNA, lncRNA, miRNA, and mRNA under XBP1 deficiency in cartilage. NcRNA plays a crucial role in the occurrence and development of diseases. To date, most of the functions of lncRNA and circRNA have not been well understood. The present study revealed that there is a variety of ncRNAs in XBP1 knockout mice, including lncRNA, circRNA, miRNA and mRNA, that are significantly differentially expressed compared with those in wild type controls. Although the results of this study need further experimental validation, the profile of these differentially expressed circRNA and lncRNA may help elucidate the occurrence and development of bone development-associated diseases and help identify prospective clinical markers.

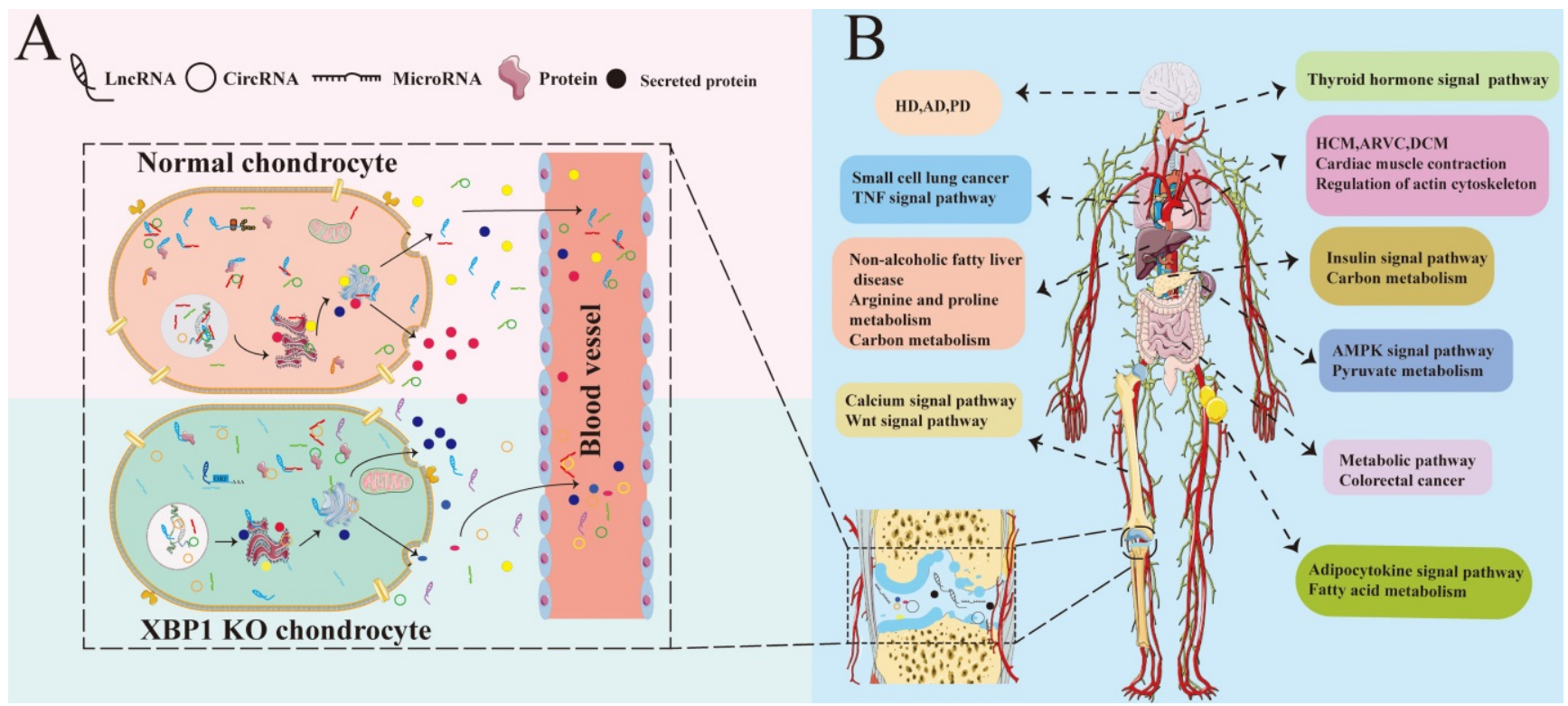

Figure 7. The model of XBP1 deficiency in cartilage is associated with various tissue diseases. (A)XBP1 deficiency in cartilage leads to differences in various of secreted factors and complete transcriptome RNA of Chondrocytes, including all kinds of ncRNAs with differential expression and regulatory profiles [63]. (B) The coordinated regulation between different tissues and organs is performed by various of secreted factors and full spectrum of transcriptome RNA, including mRNA and ncRNAs. The differential ncRNAs and secreted factors caused by XBPI deficiency in chondrocyte may cause abnormalities in different signaling pathways of different organs and tissues through blood and body fluid circulation $[64,65]$, including Wnt, insulin, AMPK, and adipocytokine signal pathway. Then these differential signal pathways lead to abnormalities in the functions of other different tissues and organs, including the nervous system, heart, bones, thyroid, and intestines. Such abnormalities, in turn, result in many kinds of different diseases. The black marks in the joint cavity represent various different ncRNAs and secreted proteins from chondrocytes. 


\section{Abbreviations}

XBP1: X-box binding protein 1; UPR: unfolded protein response; ER stress: endoplasmic reticulum stress; XBP1 CKO: XBP1conditional knockout; DE: Differentially expressed; DEGs: differential expression genes; GO: gene ontology; KEGG: Kyoto Encyclopedia of Genes and Genomes; BP: biological process; CC: cellular component; MF: molecular function; HUGO : Human Genome Organization; HCM: hypertrophic cardiomyopathy; DCM: dilated cardiomyopathy; ARVC: arrhymogenic right ventricular cardiomyopathy; PD: Parkinson's disease (PD); HD: Huntington's disease.

\section{Supplementary Material}

Supplementary figures and tables. https://www.ijbs.com/v18p0315s1.pdf

\section{Acknowledgements}

We thank Bingxiang $\mathrm{Xu}$ (Shanghai University of Sport) for his help and advice in the data analysis work. This work was aided by the National Natural Science Foundation of China (No. 81672209, No. 81871769); Chongqing Science and Technology Bureau (cstc2020jcyj-msxmX0175); Chongqing Human Resources and Social Security Bureau (2018-389).

\section{Data availability}

There are no restrictions on sharing the data. All data are available from the corresponding author upon reasonable request. Large tables (supplementary table S2, S3, S7, S8, S11, S13, S17, S18, S20, S21, S23, S24, S25, S26, S27) were deposited into GitHub with the following link https://github.com/Xiaoli-Li0921/Supplementary-Table-XBP1-Deficiency-In-Carti lage.

\section{Ethics approval}

This study was approved by the Ethics Committee of Chongqing Medical University.

\section{Author Contributions}

F.J.G., and X.L.L. designed experiments; L.L., M.T.F., and Y.Y.Y, X.Y.L, N.B.F carried out experiments; X.L.L., Y.M.P., Q.Y.T, Y.L.X, F.J.G. analyzed data; Q.B.X. provided $\mathrm{XBP} 1^{\text {flox/flox }}$ mice and give us some good advice on the interpretation of data.

Prof. Guo FJ designed the manuscript and had full access to all of the data in the study and takes responsibility for the integrity of the data and the accuracy of the data analysis. All authors approved the final manuscript prior to submission.

\section{Competing Interests}

The authors have declared that no competing interest exists.

\section{References}

1. Monemdjou R, Vasheghani F, Fahmi H, Perez G, Blati M, Taniguchi N, et al. Association of cartilage-specific deletion of peroxisome proliferator-activated receptor $\gamma$ with abnormal endochondral ossification and impaired cartilage growth and development in a murine model. Arthritis and rheumatism. 2012; 64: 1551-61.

2. Staines KA, Pollard AS, McGonnell IM, Farquharson C, Pitsillides AA. Cartilage to bone transitions in health and disease. The Journal of endocrinology. 2013; 219: R1-r12.

3. Uemura A, Oku M, Mori K, Yoshida H. Unconventional splicing of XBP1 mRNA occurs in the cytoplasm during the mammalian unfolded protein response. Journal of cell science. 2009; $122 \cdot 2877-86$.

4. Yoshida H, Oku M, Suzuki M, Mori K. pXBP1(U) encoded in XBP1 pre-mRNA negatively regulates unfolded protein response activator $\mathrm{pXBP1}(\mathrm{S})$ in mammalian ER stress response. The Journal of cell biology. 2006; 172: 565-75.

5. Todd DJ, McHeyzer-Williams LJ, Kowal C, Lee AH, Volpe BT, Diamond B, et al. XBP1 governs late events in plasma cell differentiation and is not required for antigen-specific memory B cell development. The Journal of experimental medicine. 2009; 206: 2151-9.

6. Guo FJ, Xiong Z, Han X, Liu C, Liu Y, Jiang R, et al. XBP1S, a BMP2-inducible transcription factor, accelerates endochondral bone growth by activating GEP growth factor. Journal of cellular and molecular medicine. 2014; 18: 1157-71.

7. Piróg KA, Dennis EP, Hartley CL, Jackson RM, Soul J, Schwartz JM, et al. XBP1 signalling is essential for alleviating mutant protein aggregation in ER-stress related skeletal disease. PLoS Genet. 2019; 15: e1008215.

8. Cameron TL, Gresshoff IL, Bell KM, Piróg KA, Sampurno L, Hartley CL, et al. Cartilage-specific ablation of XBP1 signaling in mouse results in a chondrodysplasia characterized by reduced chondrocyte proliferation and delayed cartilage maturation and mineralization. Osteoarthritis and cartilage. 2015; 23: 661-70

9. Panni S, Lovering RC, Porras P, Orchard S. Non-coding RNA regulatory networks. Biochimica et biophysica acta Gene regulatory mechanisms. 2020; 1863: 194417.

10. Mattick JS, Makunin IV. Non-coding RNA. Human molecular genetics. 2006; 15 Spec No 1: R17-29.

11. Hombach S, Kretz M. Non-coding RNAs: Classification, Biology and Functioning. Advances in experimental medicine and biology. 2016; 937: 3-17.

12. Esteller M. Non-coding RNAs in human disease. Nature reviews Genetics. 2011; 12: 861-74.

13. Wang X, Deng Y, Zhang G, Li C, Ding G, May HI, et al. Spliced X-box Binding Protein 1 Stimulates Adaptive Growth Through Activation of mTOR. Circulation. 2019; 140: 566-79.

14. Seal RL, Chen LL, Griffiths-Jones S, Lowe TM, Mathews MB, O'Reilly D, et al. A guide to naming human non-coding RNA genes. The EMBO journal. 2020; 39: e103777.

15. Heo JB, Lee YS, Sung S. Epigenetic regulation by long noncoding RNAs in plants. Chromosome research : an international journal on the molecular, supramolecular and evolutionary aspects of chromosome biology. 2013; 21: 685-93.

16. Gonzalez I, Munita R, Agirre E, Dittmer TA, Gysling K, Misteli T, et al. A IncRNA regulates alternative splicing via establishment of a splicing-specific chromatin signature. Nature structural \& molecular biology. 2015; 22: 370-6.

17. Li JH, Liu S, Zheng LL, Wu J, Sun WJ, Wang ZL, et al. Discovery of Protein-lncRNA Interactions by Integrating Large-Scale CLIP-Seq and RNA-Seq Datasets. Frontiers in bioengineering and biotechnology. 2014; 2: 88.

18. Tay Y, Rinn J, Pandolfi PP. The multilayered complexity of ceRNA crosstalk and competition. Nature. 2014; 505: 344-52.

19. Zhu J, Fu H, Wu Y, Zheng X. Function of lncRNAs and approaches to IncRNA-protein interactions. Science China Life sciences. 2013; 56: 876-85.

20. Mehra M, Chauhan R. Long Noncoding RNAs as a Key Player in Hepatocellular Carcinoma. Biomarkers in cancer. 2017; 9: 1179299x17737301.

21. Rühle F, Stoll M. Long non-coding RNA Databases in Cardiovascular Research. Genomics, proteomics \& bioinformatics. 2016; 14: 191-9.

22. Li X, Wu Z, Fu X, Han W. IncRNAs: insights into their function and mechanics in underlying disorders. Mutation research Reviews in mutation research. 2014; 762: 1-21.

23. Tang S, Xie Z, Wang P, Li J, Wang S, Liu W, et al. LncRNA-OG Promotes the Osteogenic Differentiation of Bone Marrow-Derived Mesenchymal Stem Cells Under the Regulation of hnRNPK. Stem cells (Dayton, Ohio). 2019; 37: 270-83.

24. Chen $X$, Yang $\mathrm{T}$, Wang $\mathrm{W}, \mathrm{Xi}$ W, Zhang $\mathrm{T}$, Li $\mathrm{O}$, et al. Circular RNAs in immune responses and immune diseases. Theranostics. 2019; 9: 588-607.

25. Haque S, Harries LW. Circular RNAs (circRNAs) in Health and Disease. Genes. 2017; 8

26. Kristensen LS, Andersen MS, Stagsted LVW, Ebbesen KK, Hansen TB, Kjems J. The biogenesis, biology and characterization of circular RNAs. Nature reviews Genetics. 2019; 20: 675-91. 
27. Liu N, Lu W, Qu X, Zhu C. LLLI promotes BMSC proliferation through circRNA_0001052/miR-124-3p. Lasers in medical science. 2021.

28. Li Z, Zhao H, Chu S, Liu X, Qu X, Li J, et al. miR-124-3p promotes BMSC osteogenesis via suppressing the GSK-3 $\beta / \beta$-catenin signaling pathway in diabetic osteoporosis rats. In vitro cellular \& developmental biology Animal. 2020; 56: 723-34.

29. Zhao G, Fu Y, Cai Z, Yu F, Gong Z, Dai R, et al. Unspliced XBP1 Confers VSMC Homeostasis and Prevents Aortic Aneurysm Formation via FoxO4 Interaction. Circulation research. 2017; 121: 1331-45.

30. Wen X, Li X, Tang Y, Tang J, Zhou S, Xie Y, et al. Chondrocyte FGFR3 Regulates Bone Mass by Inhibiting Osteogenesis. The Journal of biological chemistry. 2016; 291: 24912-21.

31. Zeng L, Li Y, Yang J, Wang G, Margariti A, Xiao Q, et al. XBP 1-Deficiency Abrogates Neointimal Lesion of Injured Vessels Via Cross Talk With the PDGF Signaling. Arteriosclerosis, thrombosis, and vascular biology. 2015; 35: 2134-44.

32. Pertea M, Kim D, Pertea GM, Leek JT, Salzberg SL. Transcript-level expression analysis of RNA-seq experiments with HISAT, StringTie and Ballgown. Nature protocols. 2016; 11: 1650-67.

33. Ghosh S, Chan CK. Analysis of RNA-Seq Data Using TopHat and Cufflinks. Methods in molecular biology (Clifton, NJ). 2016; 1374: 339-61.

34. Memczak S, Jens M, Elefsinioti A, Torti F, Krueger J, Rybak A, et al. Circular RNAs are a large class of animal RNAs with regulatory potency. Nature. 2013; 495: 333-8.

35. Gao Y, Zhang J, Zhao F. Circular RNA identification based on multiple seed matching. Briefings in bioinformatics. 2018; 19: 803-10.

36. Gao G, Xie A, Zhang J, Herman AM, Jeong EM, Gu L, et al. Unfolded protein response regulates cardiac sodium current in systolic human heart failure. Circulation Arrhythmia and electrophysiology. 2013; 6: 1018-24.

37. Langmead B, Trapnell C, Pop M, Salzberg SL. Ultrafast and memory-efficient alignment of short DNA sequences to the human genome. Genome biology. 2009; 10: R25.

38. Friedländer MR, Mackowiak SD, Li N, Chen W, Rajewsky N. miRDeep2 accurately identifies known and hundreds of novel microRNA genes in seven animal clades. Nucleic acids research. 2012; 40: 37-52

39. Wen M, Shen $Y$, Shi S, Tang T. miREvo: an integrative microRNA evolutionary analysis platform for next-generation sequencing experiments. BMC bioinformatics. 2012; 13: 140

40. Fan RF, Cao CY, Chen MH, Shi QX, Xu SW. Gga-let-7f-3p promotes apoptosis in selenium deficiency-induced skeletal muscle by targeting selenoprotein $\mathrm{K}$. Metallomics : integrated biometal science. 2018; 10: 941-52.

41. Zhou L, Chen J, Li Z, Li X, Hu X, Huang Y, et al. Integrated profiling of microRNAs and mRNAs: microRNAs located on Xq27.3 associate with clear cell renal cell carcinoma. PloS one. 2010; 5: e15224.

42. Mortazavi A, Williams BA, McCue K, Schaeffer L, Wold B. Mapping and quantifying mammalian transcriptomes by RNA-Seq. Nature methods. 2008; 5: 621-8.

43. Robinson MD, McCarthy DJ, Smyth GK. edgeR: a Bioconductor package for differential expression analysis of digital gene expression data. Bioinformatics (Oxford, England). 2010; 26: 139-40.

44. Young MD, Wakefield MJ, Smyth GK, Oshlack A. Gene ontology analysis for RNA-seq: accounting for selection bias. Genome biology. 2010; 11: R14.

45. Kanehisa M, Araki M, Goto S, Hattori M, Hirakawa M, Itoh M, et al. KEGG for linking genomes to life and the environment. Nucleic acids research. 2008; 36: D480-4.

46. Guo LL, Song CH, Wang P, Dai LP, Zhang JY, Wang KJ. Competing endogenous RNA networks and gastric cancer. World journal of gastroenterology. 2015; 21: 11680-7.

47. Li R, Qu H, Wang S, Wei J, Zhang L, Ma R, et al. GDCRNATools: an $\mathrm{R} /$ Bioconductor package for integrative analysis of IncRNA, miRNA and mRNA data in GDC. Bioinformatics (Oxford, England). 2018; 34: 2515-7.

48. Hansen TB, Jensen TI, Clausen BH, Bramsen JB, Finsen B, Damgaard CK, et al. Natural RNA circles function as efficient microRNA sponges. Nature. 2013; 495: 384-8.

49. Lee JW, Kim YH, Kim SH, Han SH, Hahn SB. Chondrogenic differentiation of mesenchymal stem cells and its clinical applications. Yonsei medical journal. 2004; 45 Suppl: 41-7.

50. Guo F, Lin EA, Liu P, Lin J, Liu C. XBP1U inhibits the XBP1S-mediated upregulation of the iNOS gene expression in mammalian ER stress response. Cellular signalling. 2010; 22: 1818-28.

51. Groff AF, Sanchez-Gomez DB, Soruco MML, Gerhardinger C, Barutcu AR, Li E, et al. In Vivo Characterization of Linc-p21 Reveals Functional cis-Regulatory DNA Elements. Cell reports. 2016; 16: 2178-86.

52. Li S, Sun X, Miao S, Lu T, Wang Y, Liu J, et al. hsa_circ_0000729, a potential prognostic biomarker in lung adenocarcinoma. Thoracic cancer. 2018; 9: 224-30.

53. Zeng $\mathrm{Y}$, Wang $\mathrm{KX}, \mathrm{Xu} \mathrm{H}$, Hong $\mathrm{Y}$. Integrative miRNA analysis identifies hsa-miR-3154, hsa-miR-7-3, and hsa-miR-600 as potential prognostic biomarker for cervical cancer. Journal of cellular biochemistry. 2018; 119: 1558-66.

54. Beermann J, Piccoli MT, Viereck J, Thum T. Non-coding RNAs in Development and Disease: Background, Mechanisms, and Therapeutic Approaches. Physiological reviews. 2016; 96: 1297-325.
55. Wu Y, Lu X, Shen B, Zeng $Y$. The Therapeutic Potential and Role of miRNA, IncRNA, and circRNA in Osteoarthritis. Current gene therapy. 2019; 19: 255-63.

56. Abbasifard M, Kamiab Z, Bagheri-Hosseinabadi Z, Sadeghi I. The role and function of long non-coding RNAs in osteoarthritis. Experimental and molecular pathology. 2020; 114: 104407.

57. Mercer TR, Dinger ME, Mattick JS. Long non-coding RNAs: insights into functions. Nature reviews Genetics. 2009; 10: 155-9.

58. Han Y, You X, Xing W, Zhang Z, Zou W. Paracrine and endocrine actions of bone-the functions of secretory proteins from osteoblasts, osteocytes, and osteoclasts. Bone research. 2018; 6:16.

59. Sanchez C, Bay-Jensen AC, Pap T, Dvir-Ginzberg M, Quasnichka H, Barrett-Jolley R, et al. Chondrocyte secretome: a source of novel insights and exploratory biomarkers of osteoarthritis. Osteoarthritis and cartilage. 2017; 25 : 1199-209.

60. Gu W, Shi J, Liu H, Zhang X, Zhou JJ, Li M, et al. Peripheral blood non-canonical small non-coding RNAs as novel biomarkers in lung cancer. Molecular cancer. 2020; 19: 159.

61. Fanale D, Taverna S, Russo A, Bazan V. Circular RNA in Exosomes. Advances in experimental medicine and biology. 2018; 1087: 109-17.

62. Perez de Acha O, Rossi M, Gorospe M. Circular RNAs in Blood Malignancies. Frontiers in molecular biosciences. 2020; 7: 109.

63. Sun H, Peng G, Ning X, Wang J, Yang H, Deng J. Emerging roles of long noncoding RNA in chondrogenesis, osteogenesis, and osteoarthritis. American journal of translational research. 2019; 11: 16-30.

64. Dykstra-Aiello C, Jickling GC, Ander BP, Shroff N, Zhan X, Liu D, et al. Altered Expression of Long Noncoding RNAs in Blood After Ischemic Stroke and Proximity to Putative Stroke Risk Loci. Stroke. 2016; 47: 2896-903.

65. He J, Wu F, Han Z, Hu M, Lin W, Li Y, et al. Biomarkers (mRNAs and Non-Coding RNAs) for the Diagnosis and Prognosis of Colorectal Cancer From the Body Fluid to Tissue Level. Frontiers in oncology. 2021; 11: 632834. 